\title{
Clinicopathological characteristics, diagnosis, treatment, and outcomes of primary gastric adenosquamous carcinoma
}

\author{
Haining Chen ${ }^{1+}$, Chaoyong Shen ${ }^{1 \dagger}$, Rui Yin ${ }^{2}$, Yuan Yinn ${ }^{1}$, Jiaju Chen ${ }^{1}$, Luyin Han ${ }^{3}$, Bo Zhang ${ }^{1 *}$, Zhixin Chen ${ }^{1}$
} and Jiaping Chen ${ }^{1}$

\begin{abstract}
Background: Primary gastric adenosquamous carcinoma (ASC) is a rare subset of ASC. This study aims to investigate the clinicopathological features, diagnosis, treatment, and outcomes of primary gastric ASC.

Methods: The medical records of 13 consecutive patients with primary gastric ASC between January 2010 and July 2014 from a single institutional database were reviewed.

Results: Male predominance was observed (M/F =10/3) among the patients, and their median age was 62 years (range: 43 to 79 years). The primary lesions were most often found in the upper third of the stomach, with a median tumor size of $5 \mathrm{~cm}$ (range: $2.25 \mathrm{~cm}$ to $10.5 \mathrm{~cm}$ ). Ten patients underwent radical resections (R0 resection, 76.9\%), while three patients had palliative resections (R1/R2 resection, 23.1\%). Twelve patients had lymph node metastasis at the time of surgery. Adenocarcinoma and squamous cell carcinoma components in lymph node were found in eight and two cases, respectively, while two patients had both squamous cell carcinoma and adenocarcinoma components. In terms of the TNM staging system, stages IIB, IIIA, III, IIIC, and IV were detected in 2 (15.4\%), 2 (15.4\%), 1 (7.7\%), 5 (38.5\%), and 3 (23.1\%) patients, respectively. The median follow-up period was 22 months (range: 5 to 52 months); during which, four patients were still alive and eight patients died because of tumor progression. The 1-, 2-, and 3-year survival rates were 76.9\%, 46.2\%, and 15.4\%, respectively.
\end{abstract}

Conclusions: Primary gastric ASC has a very poor prognosis, and both squamous cell carcinoma and adenocarcinoma components have distant metastasis potential.

Keywords: Adenosquamous carcinoma, Clinicopathologic, Diagnosis, Prognosis, Stomach neoplasms

\section{Background}

Adenosquamous carcinoma (ASC) is a rare entity that occurs throughout the entire alimentary tract [1-3]. The incidence of primary gastric ASC is uncommon, accounting for less than $1 \%$ of the total gastric carcinomas worldwide $[4,5]$ and mostly affecting Asians. Primary gastric ASC exhibits early tumor progression and poorer prognosis than the typical gastric adenocarcinoma [4]. The biological behavior of ASC is usually determined by the adenocarcinoma component [6,7]. Patients with

\footnotetext{
*Correspondence: hxwcwk@126.com

${ }^{\dagger}$ Equal contributors

'Department of Gastrointestinal Surgery, West China Hospital, Sichuan University, 37 Guoxue Alley, Chengdu 610041, Sichuan, China

Full list of author information is available at the end of the article
}

gastric ASC have variable clinical symptoms, some of which are identical to those of other types of gastric tumors.

Given the rarity of these carcinomas, most literatures are based on case reports [2,4,5,7-11]. The histogenesis of these tumors has been debated considerably, and clinical therapies and prognosis of ASC have not been well established to date [12]. Therefore, in the current study, we incorporated the data of 13 consecutive patients with primary gastric ASC who underwent surgery at a single medical institution. We investigated the clinicopathological features, diagnosis, treatment, and survival outcomes of these patients to contribute to deeper knowledge on this tumor and provide additional assistance for ASC management. 


\section{Methods}

\section{Patient selection}

Thirteen patients who were surgically treated and pathologically diagnosed with primary gastric ASC at the Department of Gastrointestinal Surgery, West China Hospital, Sichuan University from January 2010 to July 2014 were retrospectively identified. Contrast-enhanced computed tomography scan of the chest and abdomen, electronic gastroscopy, lung function, renal and liver function, and others were routinely performed preoperatively. All surgical samples were reviewed by a senior pathologist from our institution. ASC diagnosis was confirmed when the characteristics of coexistence of squamous cell carcinoma (SC) and adenocarcinoma components (AC) were identified, with SC accounting for at least $20 \%$ to $25 \%[13,14]$. Patients diagnosed with nongastric ASC having incomplete medical records and did not attend the follow-up examination were excluded from this study. The Institutional Review Board and Ethics Committee of the West China Hospital of Sichuan University informed that an ethical review was not needed for this retrospective study.

\section{Surgery and immunohistochemical examination}

All patients underwent laparotomy with general anesthesia; surgical procedures were performed according to the criteria of the Japanese Gastric Cancer Association [13], including subtotal gastrectomy or total gastrectomy and D2 lymphadenectomy or greater D2 lymphadenectomy. Operation consent was obtained from each patient who underwent surgical resection. Two patients underwent proximal gastrectomy with gastric tube reconstruction. The specimens for histological examination were fixed in $10 \%$ buffered formalin and stained immunohistochemically for carcinoembryonic antigen (CEA), cytokeratin (CK) 5/6/7, p63, chromogranin A (CgA), and synaptophysin (Syn).

\section{Data collection and follow-up}

The parameters collected from the medical records and pathological reports included demographic data, clinical manifestation at the time of diagnosis, hospital stay, surgical data, tumor size and location, pathological data (Borrmann types, depth of invasion, lymphatic metastasis, TNM stages, and so on.), immunohistochemical staining, postoperative tumor recurrence, and so on. Tumor TNM stages were graded according to the criteria of the Seventh Edition of the American Joint Committee on Cancer Staging Manual [15]. Overall survival time was calculated from the time of surgery to death or until the last followup. Follow-up was conducted through office visit, telephone, or outpatient clinic visit from September 2014 to October 2014.

\section{Results}

Patient characteristics

The clinicopathological data of the ASC patients are summarized in Tables 1 and 2. This entire cohort is comprised of 10 males and 3 females, with a malefemale ratio of $3.3: 1$. The ages of the patients ranged from 43 to 79 years (median: 62 years). A preoperative biopsy showed ASC in only two patients. The primary lesions were mostly found in the upper third of stomach, with a median tumor size of $5 \mathrm{~cm}$ (range: 2.25 to $10.5 \mathrm{~cm})$. Borrmann types I, II, and III carcinoma were observed in 1 (7.7\%), 6 (46.2\%), and 6 (46.2\%) cases, respectively. The clinical symptoms were identical to those of other types of gastric tumors, with epigastric pain, dysphagia, and acid regurgitation as the main clinical manifestations. The mean hospital stay length was $19.3 \pm$ 6.1 days. Two patients (numbers 2 and 5) had type 2 diabetes mellitus, while one patient (number 5) was confirmed with liver cirrhosis during the operation.

\section{Surgical and adjuvant treatment}

All 13 ASC patients were surgically treated through abdominal approach, including D2 lymphadenectomy or greater D2 lymphadenectomy. Ten patients underwent radical resections ( $\mathrm{R} 0$ resection, $76.9 \%$ ), while the other three patients had palliative resections (23.1\%), including multiple liver metastasis (number 11), liver metastasis with cirrhosis (number 5), and involvement of pancreas/ spleen (number 2). Total, proximal, and distal gastrectomy was conducted in 3,6 , and 4 cases, respectively. Two patients underwent gastric tube reconstruction after proximal gastrectomy. In terms of the TNM staging system, stages IIB, IIIA, IIIB, IIIC, and IV were detected in 2 (15.4\%), 2 (15.4\%), 1 (7.7\%), 5 (38.5\%), and $3(23.1 \%)$ patients, respectively. Adjuvant therapies were routinely recommended for patients with palliative resection, and stage II, III, or IV. Chemotherapy regimens for squamous carcinoma should be conducted when SC component becomes a dominant in tumor tissue. A total of six patients received adjuvant therapy. Three patients were treated with chemotherapy of SOX regimen for five cycles (numbers 1,9 , and 11), while the other three patients received FOLFIRI for three cycles (number 5), FOLFOX for seven cycles (number 4), and Gimeracil and Oteracil Porassium capsules for four cycles (number 12). Two (numbers 1 and 9) of the six patients also received abdominal radiotherapy (GTV 60.2GY/28f, CTV 50.4GY/28f).

\section{Pathological and immunohistochemical examination}

Table 2 shows the pathological characteristics of the 13 primary gastric ASC patients. ASC was characterized as a mixture of SC and AC (Figure 1). Most squamous cell carcinoma components had the characteristics of individual cell keratinization, keratin pearl, or intercellular 
Table 1 Clinicopathological characteristics and demographical data of 13 patients with primary gastric ASC

\begin{tabular}{|c|c|c|c|c|c|c|c|c|c|}
\hline $\begin{array}{l}\text { Case } \\
\text { number }\end{array}$ & Gender & Age & Site & Size $(\mathrm{cm})$ & $\begin{array}{l}\text { Borrmann } \\
\text { type }\end{array}$ & $\begin{array}{l}\text { Hospital } \\
\text { stay (days) }\end{array}$ & Operation & Postoperative complication & Adjuvant therapy \\
\hline 1 & M & 51 & U & $2.5^{*} 2$ & $\|$ & 29 & Radical & Abdominal infection & Yes \\
\hline 2 & $\mathrm{~F}$ & 66 & M & $11 * 10$ & III & 28 & Palliative & - & No \\
\hline 3 & M & 63 & U & $7^{* 6} 6$ & III & 21 & Radical & - & No \\
\hline 4 & M & 43 & $\mathrm{~L}$ & $6 * 5$ & $\|$ & 11 & Radical & Pulmonary infection & Yes \\
\hline 5 & M & 79 & U & $7 * 5$ & III & 27 & Palliative & lleus & Yes \\
\hline 6 & M & 62 & U & $8 * 4$ & $\|$ & 21 & Radical & - & No \\
\hline 7 & M & 47 & $L$ & $5 * 5$ & $\|$ & 11 & Radical & - & No \\
\hline 8 & $\mathrm{~F}$ & 70 & U & $4^{*} 4$ & । & 18 & Radical & - & No \\
\hline 9 & M & 63 & L & $5 * 5$ & III & 17 & Radical & - & Yes \\
\hline 10 & M & 73 & $L$ & $4 * 3$ & III & 13 & Radical & Digestive bleeding & No \\
\hline 11 & M & 56 & U & $6 * 3$ & $\|$ & 16 & Palliative & - & Yes \\
\hline 12 & $\mathrm{~F}$ & 50 & M & $3 * 3$ & $\|$ & 22 & Radical & - & Yes \\
\hline 13 & M & 61 & U & $5.5^{*} 4$ & III & 17 & Radical & - & No \\
\hline
\end{tabular}

Abbreviations: ASC adenosquamous carcinoma, $M$ male, $F$ female, $U$ upper third of stomach, $M$ middle third of stomach, $L$ lower third of stomach.

bridge, among others. Seven patients underwent immunohistochemical examination. Both CEA and CK7 were positive in all $\mathrm{AC}$ of tumor or $\mathrm{AC}$ of metastatic lymph node; however, SC with these two antibodies were weakly positive in one and three cases, respectively. P63 expression was positive in the squamous cell carcinoma component in six patients but negative in adenocarcinoma components. No patient had positive expression of CgA and Syn. The tumor invaded the visceral peritoneum (T4a) and adjacent tissues (T4b) in six and three cases, respectively. A total of 359 lymph nodes were moved; among which, 106 had metastases. Adenocarcinoma and squamous cell carcinoma components in lymph node were found in eight and two cases, respectively, while two patients had both squamous cell carcinoma and adenocarcinoma components (Figure 2).

\section{Survival outcomes}

Follow-up was carried out in all 13 patients, with a median of 22 months (range: 5 to 52 months). Four patients were still alive, whereas eight cases died because of tumor progression. Moreover, one patient (number 13) died because of chronic respiratory failure. All but two patients with TNM stages IIIA and IIB experienced tumor recurrence and/or metastasis, with a median time of progression of 7 months. The most common metastases were detected in the liver of five patients, followed by peritoneum in three cases. Meanwhile, one patient

Table 2 Pathological characteristics and survival outcomes of 13 patients with primary gastric ASC

\begin{tabular}{|c|c|c|c|c|c|c|c|c|}
\hline $\begin{array}{l}\text { Case } \\
\text { number }\end{array}$ & $\begin{array}{l}\text { TNM } \\
\text { stage }\end{array}$ & $\begin{array}{l}\text { Depth of } \\
\text { invasion }\end{array}$ & $\begin{array}{l}\text { Metastasis } \\
\text { rate of } L N\end{array}$ & Distant metastasis & $\begin{array}{l}\text { Proportion } \\
\text { of SCC }\end{array}$ & $\begin{array}{l}\text { Metastatic } \\
\text { components in LN }\end{array}$ & $\begin{array}{l}\text { Recurrence/metastasis } \\
\text { (months) }\end{array}$ & $\begin{array}{l}\text { Outcomes/period } \\
\text { (months) }\end{array}$ \\
\hline 1 & IIIA & T4a & $2 / 24$ & MO & $40 \%$ & $A C$ & Liver (7) & Death (26) \\
\hline 2 & IV & $\mathrm{T} 4 \mathrm{~b}$ & $4 / 29$ & Spleen/pancreas & $25 \%$ & $A C$ & Peritoneal cavity (0) & Death (5) \\
\hline 3 & $\| \mathrm{IIC}$ & $\mathrm{T} 4 \mathrm{a}$ & $16 / 63$ & MO & $65 \%$ & $A C$ and $S C$ & Anastomosis (2) & Death (7) \\
\hline 4 & $\| \mathrm{II}$ & T3 & $8 / 32$ & MO & $25 \%$ & $A C$ & Peritoneum (17) & Death (31) \\
\hline 5 & IV & $\mathrm{T} 4 \mathrm{~b}$ & $4 / 27$ & Liver & $35 \%$ & $A C$ & Liver (0) & Alive (15) \\
\hline 6 & $\| \mathrm{B}$ & $\mathrm{T} 3$ & $0 / 12$ & MO & $40 \%$ & Absent & Peritoneum (21) & Death (34) \\
\hline 7 & $\mathrm{IIIC}$ & T4a & $27 / 32$ & MO & $30 \%$ & $\mathrm{AC}$ and $\mathrm{SC}$ & Lung (13) & Death (18) \\
\hline 8 & IIIA & T4a & $2 / 19$ & MO & $75 \%$ & $A C$ & Absent (-) & Alive (32) \\
\hline 9 & IIIC & $\mathrm{T} 4 \mathrm{a}$ & $11 / 19$ & MO & $60 \%$ & SC & Liver (10) & Alive (41) \\
\hline 10 & $\| B$ & $\mathrm{~T} 2$ & $4 / 21$ & MO & $20 \%$ & $A C$ & Absent (-) & Alive (52) \\
\hline 11 & IV & T3 & $9 / 29$ & Liver & $35 \%$ & $A C$ & Liver (0) & Death (13) \\
\hline 12 & $\| I C$ & $\mathrm{~T} 4 \mathrm{~b}$ & $8 / 24$ & MO & $25 \%$ & SC & Liver (9) & Death (22) \\
\hline 13 & IIIC & T4a & $11 / 28$ & MO & $45 \%$ & $A C$ & Peritoneum (5) & Death (11) \\
\hline
\end{tabular}

Abbreviations: ASC adenosquamous carcinoma, LN lymph nodes, AC adenocarcinoma components, SC squamous cell carcinoma components. 

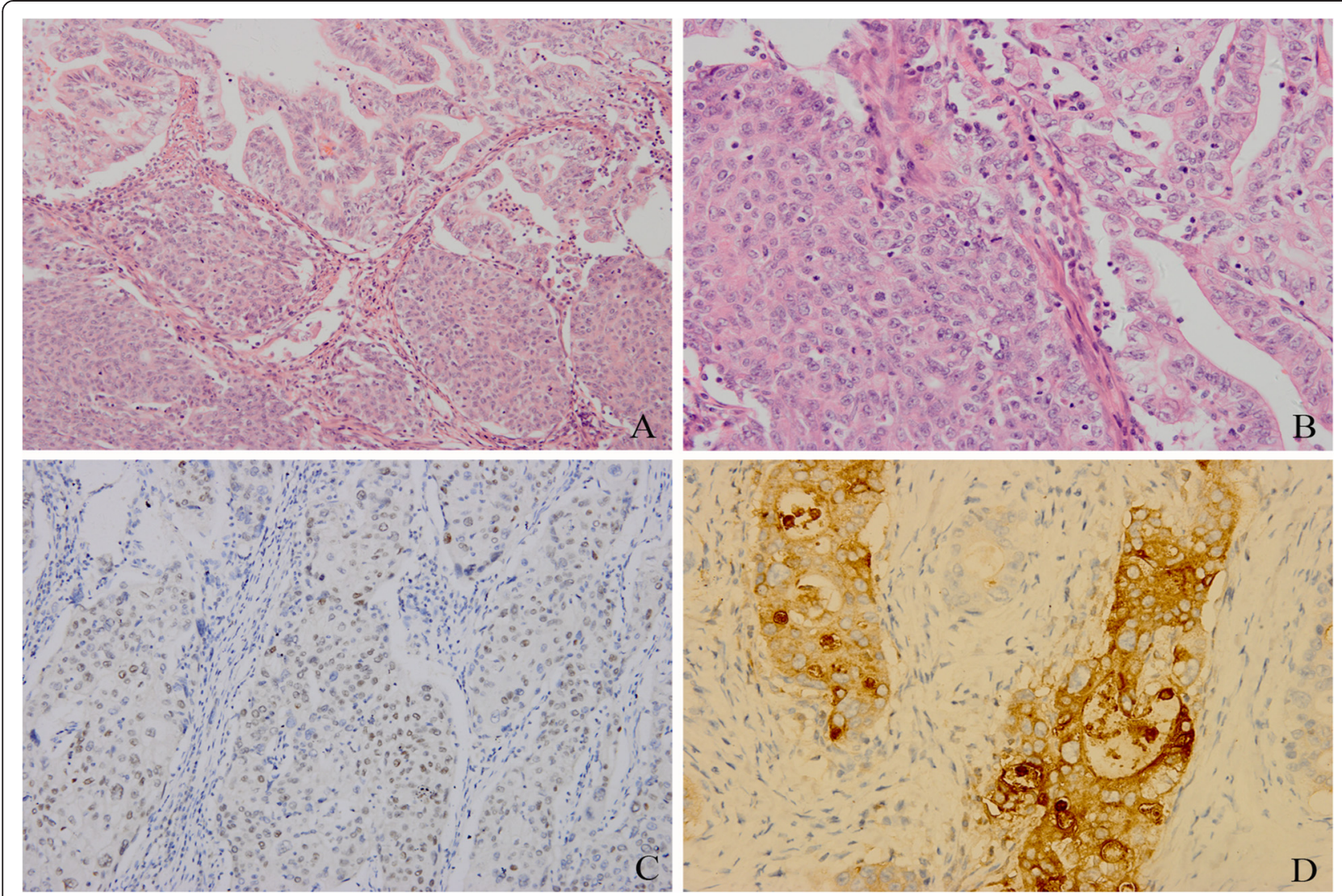

Figure 1 ASC characterized as a mixture of SC and AC. Both squamous cell carcinoma and adenocarcinoma components were found

(A: hematoxylin-eosin staining, $\times 200$; B: hematoxylin-eosin staining, $\times 400)$; Immunohistochemistry of p63 protein that was positive in the squamous cell carcinoma area $(\mathbf{C}: \times 400)$, and positive staining for adenocarcinoma cell with CEA (D: $\times 400)$.
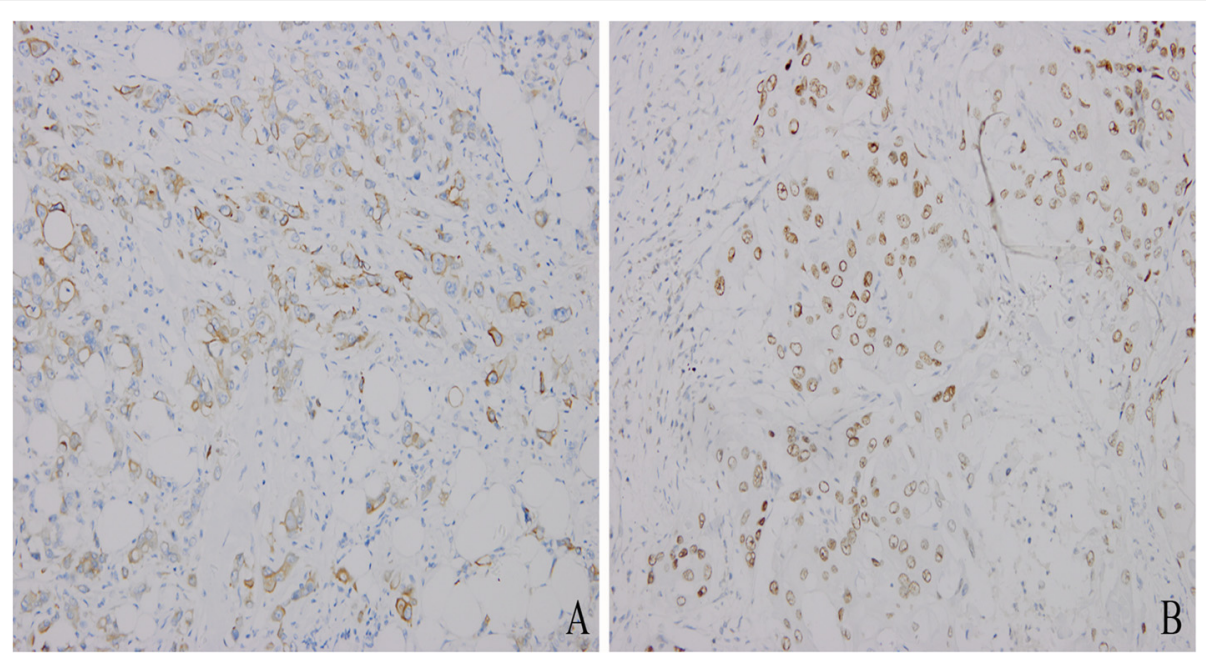

Figure 2 Squamous cell carcinoma and adenocarcinoma components were found in the lymph node metastasis in the same patient.

Adenocarcinoma component, positive for CK7 (A: ×400); and squamous component, positive for p63 (B: $\times 400)$. 
(number 9) with liver metastases underwent radiofrequency ablation. The median overall survival time and time to progression were 22 and 9 months, respectively. A trend for better survival was observed in patients treated with adjuvant therapy (median survival time: 24 months vs. 18 months). The 1-, 2-, and 3-year survival rates were $76.9 \%, 46.2 \%$, and $15.4 \%$, respectively. Given the limited sample size, analysis of the relevant prognosis factors of ASC was not attempted.

\section{Discussion}

Adenocarcinoma is the most common malignant tumor of the stomach; primary gastric ASC is a rare clinical entity. ASC of the stomach has gained a great deal of interests because of its unsolved histogenesis; its clinicopathological and immunohistochemical characteristics are poorly understood $[7,13]$. Rolleston and Trevor first reported on gastric ASC in 1905. Notably, the majority of patients with ASC were diagnosed at an advanced stage, with high-grade malignancy and poor prognosis $[2,4]$. ASC occurrence is more frequent in the proximal stomach among middle-aged and aged populations, as well as among Asian men [1,5,16]. In the current study, the male to female ratio was $3.3: 1$. Occurrence is mainly observed in the upper third of the stomach among patients with median age of 62 years, which is in agreement with previous reports. The clinical presentations of gastric ASC can be variable but are basically identical to those of other types of gastric cancer, and presented mainly as epigastric pain $[4,7]$.

According to the World Health Organization classification, ASC consists of a mixture of adenocarcinoma and squamous cell carcinoma components. However, the histogenesis of SC remains controversial. The following hypotheses have been proposed concerning its most likely origin $[1,4,8,17-19]$ : 1 . Foci of heterotopic squamous epithelia in the gastric mucosa; 2. Stem cell of the gastric mucosa that differentiated toward both cellular lines; 3. Metaplastic transformation of an adenocarcinoma or benign squamous gastric epithelia; 4 . Endothelial cells differentiated toward squamous elements; and 5 . Collision of an adenocarcinoma and a squamous cell carcinoma. Currently, the hypothesis that SCs are derived from adenocarcinoma is favored by many scholars. ASC has one obvious transition area between the two components and adjoined adenocarcinoma components. The positive expression of CK7 and CEA in SC from the available data supports the hypothesis that $\mathrm{SC}$ originated from adenocarcinoma [1,19]. Moreover, SC in ASC may differentiate into those that resemble pure squamous cell carcinoma or those with totally different biological behaviors [17]. In this study, weak positive expression of CEA and CK7 in SC were observed with one and three cases, respectively. Hence, more studies are required to confirm the histogenesis of gastric ASC.

Macroscopically, the majority of ASCs observed are Borrmann types II and III, whereas types I and IV are rare $[17,20]$. Diagnosed ASC is usually accompanied by distant metastasis, such as peritoneal dissemination, lymph node metastasis, and liver metastasis [4-6]. As shown in the present series, the most common site of metastasis is the liver, followed by peritoneal dissemination. We also found that both SC and AC have the potential for distant metastasis, similar to those reported in other publications $[1,17]$. ASC is usually found deep into the muscular layer, with more aggressive clinicopathological characteristics. The findings in this study show that the tumor invaded the visceral peritoneum (T4a) or adjacent tissues (T4b) in six and three cases, respectively. Most patients were diagnosed at more advanced stages. A noteworthy standpoint was raised by Bansal et al. [7], who considered that the biological behaviors may be determined by the AC in ASC of the stomach. Meanwhile, several studies have shown that the biological behaviors of ASC are usually similar to those of an aggressive adenocarcinoma [6,18,21]. However, a convincing conclusion cannot be reached based on a case report or observation with a small number of samples.

No standard treatment for primary gastric ASC has been established because of its rarity. At present, complete surgical resection to achieve R0, including resetting the proximity of organs or directly surrounding normal tissues as necessary, remains the main curative method for all malignant tumors. Regrettably, the therapeutic outcomes of ASC are unsatisfactory, and most patients exhibit a very rapid deteriorating course within a few months postoperatively $[4,7]$. Patients who received adjuvant chemotherapy had better outcomes. S-1 monotherapy, cisplatin, fluoropyrimidine, docetaxel, and irinotecan reportedly improve survival times, but still with poor prognosis $[1,5,10,22]$. Squamous carcinoma is sensitive to radiotherapy; hence, postoperative radiotherapy can also be used as one of the comprehensive treatments for ASC. As described previously, patients with cervical ASC derive relatively better survival benefits from adjuvant radiotherapy $[23,24]$. In our study, a trend for better survival in patients treated with adjuvant therapy was observed (median survival time: 24 months vs. 18 months). Nevertheless, in an effort to gain confirmation of this finding and establish the standard therapeutic approaches, more data concerning gastric ASC should be collected.

The prognosis of ASC has been discussed in previous reports. ASC is more aggressive than pure adenocarcinoma. The median survival time of gastric ASC ranges from 12 to 22 months, and the 5 -year survival rate is $10 \%[1,5]$. A similar survival result of esophageal ASC was reported by Chen et al [14]. Their study showed 
that the median survival time was 21 months, and the 1-, $3-$, and 5-year overall survival rates were $67.5 \%, 29.4 \%$, and $22.9 \%$, respectively. By contrast, cervical ASC patients seem to have a relatively better outcome [23]. In the current study, the median overall survival time and time to progression were 22 and 9 months, respectively. Several factors affect the prognosis of tumors, such as TNM stage, tumor size, site, and surgical margin. A study by Lee $e t a l$. [17] showed that p53 protein over-expression and high Ki-67 labeling index maybe predictors of poor prognosis. Given the limited sample size in our study, we did not attempt to analyze the independent prognostic factors regarding primary gastric ASC.

\section{Conclusions}

Primary gastric ASC is a rare entity with poor prognosis. Surgical resection is still the gold standard treatment for primary gastric ASC, although the rate of tumor recurrence/metastasis remains high even after radical resection. Adjuvant therapy (chemotherapy and/or radiotherapy) can improve survival time. Large-sample investigations are needed to determine the prognostic factors and find new therapeutic approaches to achieve better prognosis.

\section{Abbreviations}

AC: adenocarcinoma components; ASC: adenosquamous carcinoma; CEA: carcinoembryonic antigen; CgA: chromogranin A; CK: cytokeratin; SC: squamous cell carcinoma components; Syn: synaptophysin.

\section{Competing interests}

The authors declare that they have no competing interests.

\section{Authors' contributions}

$\mathrm{ZB}, \mathrm{CZX}$, and CJP conceived the study and participated in its design and coordination. CHN, SCY, YY, CJJ, and HLY helped to collect data. CHN and SCY drafted the manuscript. YR helped to review the immunohistochemical data. ZB helped in revising the paper critically for important intellectual content. All authors read and approved the final manuscript.

\section{Acknowledgements}

We would like to thank the Department of Gastrointestinal Surgery and Pathology, West China Hospital, who generously provided assistance in the collection of data throughout the duration of the study.

\section{Author details}

'Department of Gastrointestinal Surgery, West China Hospital, Sichuan University, 37 Guoxue Alley, Chengdu 610041, Sichuan, China. ²Department of Pathology, West China Hospital, Sichuan University, 37 Guoxue Alley, Chengdu 610041, Sichuan, China. Intensive Care Unit, West China Hospital, Sichuan University, 37 Guoxue Alley, Chengdu 610041, China.

Received: 12 January 2015 Accepted: 16 March 2015

Published online: 02 April 2015

\section{References}

1. Saito S, Hosoya Y, Morishima K, Ui T, Haruta H, Kurashina K, et al. A clinicopathological and immunohistochemical study of gastric cancer with squamous cell carcinoma components: a clinically aggressive tumor. J Dig Dis. 2012;13(8):407-13.

2. Choi JW, Park HU. Adenosquamous carcinoma of the ascending colon: a case report and review of the literature. Ann Coloproctol. 2013;29(2):83-6.

3. Hong SM, Kim MJ, Jang KT, Yoon GS, Cho H, Frierson HF, et al. Adenosquamous carcinoma of extrahepatic bile duct: clinicopathologic study of 12 cases. Int J Clin Exp Pathol. 2008;1 (2):147-56.
4. Faria GR, Eloy C, Preto JR, Costa EL, Almeida T, Barbosa J, et al. Primary gastric adenosquamous carcinoma in a Caucasian woman: a case report. J Med Case Rep. 2010:4:351.

5. Ebi M, Shimura T, Yamada S, Hirata Y, Tsukamoto H, Okamoto Y, et al. A patient with gastric adenosquamous carcinoma with intraperitoneal free cancer cells who remained recurrence-free with postoperative S-1 chemotherapy. Intern Med. 2012;51(22):3125-9.

6. Kim YS, Heo WS, Chae KH, Gang YS, Jung JH, Kim SH, et al. Clinicopathologic features and differences of p53 and Ki-67 expression in adenosquamous and squamous cell carcinomas of the stomach. Korean J Gastroenterol. 2006;47(6):425-31.

7. Bansal RK, Sharma P, Kaur P, Arora A. Primary gastric adenosquamous carcinoma in an Indian male. Indian J Pathol Microbiol. 2013;56(4):416-8.

8. Mori E, Watanabe A, Maekawa S, Itasaka H, Maeda T, Yao T. Adenosquamous carcinoma of the remnant stomach: report of a case. Surg Today. 2000;30(7):643-6.

9. Yoshida K, Manabe T, Tsunoda T, Kimoto M, Tadaoka Y, Shimizu M. Early gastric cancer of adenosquamous carcinoma type: report of a case and review of literature. Jpn J Clin Oncol. 1996;26(4):252-7.

10. Hirano M, Ozamoto Y, Ichinose M, Togawa T, Takao N, Mizumoto A, et al. A case of gastric adenosquamous carcinoma with peritoneal dissemination in which treatment with S-1 plus paclitaxel therapy resulted in improved longterm survival. Gan To Kagaku Ryoho. 2014;41(7):885-8.

11. Ajoodhea $H$, Zhang RC, Xu XW, Jin WW, Chen $K$, He YT, et al. Fever as a first manifestation of advanced gastric adenosquamous carcinoma: a case report. World J Gastroenterol. 2014;20(29):10193-201.

12. Tohma T, Yamamoto $Y$, Seki $Y$, Takaishi S, Sakuma Y, Funami $Y$, et al. Weekly paclitaxel therapy is effective for gastric adenosquamous carcinoma: a case report. Hepatogastroenterology. 2009;56(90):568-70.

13. Japanese Gastric Cancer Association. Japanese classification of gastric carcinoma: ${ }^{\text {rd }}$ English edition. Gastric Cancer. 2011;14(2):101-12.

14. Chen SB, Weng HR, Wang G, Yang JS, Yang WP, Liu DT, et al. Primary adenosquamous carcinoma of the esophagus. World J Gastroenterol. 2013;19(45):8382-90.

15. Washington K. 7th Edition of the AJCC Cancer Staging Manual: Stomach. Ann Surg Oncol. 2010;17(12):3077-9.

16. Quan J, Zhang R, Liang H, Li F, Liu H. The clinicopathologic and prognostic analysis of adenosquamous and squamous cell carcinoma of the stomach. Am Surg. 2013;79(5):E206-208.

17. Lee WA, Woo DK, Kim Yl, Kim WH. p53, p16 and RB expression in adenosquamous and squamous cell carcinomas of the stomach. Pathol Res Pract. 1999;195(11):747-52.

18. Toyota N, Minagi S, Takeuchi T, Sadamitsu N. Adenosquamous carcinoma of the stomach associated with separate early gastric cancer (type IIc). J Gastroenterol. 1996;31(1):105-8.

19. He YT, Wang XJ, Gong J, Chen N, Zhou Q. Primary adenosquamous carcinoma of the jejunum. Pathol Int. 2005;55(9):590-5.

20. Endo K, Kohnoe S, Okamura T, Haraguchi M, Adachi E, Toh Y, et al. Gastric adenosquamous carcinoma producing granulocyte-colony stimulating factor. Gastric Cancer. 2005;8(3):173-7.

21. Manna ED, Seixas AA, de Araújo RP, Ferro MC. Primary adenosquamous carcinoma of the stomach. Rev Assoc Med Bras. 1998;44(2):152-4.

22. Ishiguro A, Takahata T, Hirose K, Matsumoto Y, Tanaka S, Suzuki K, et al. A case of gastric adenosquamous carcinoma successfully treated with second-line chemotherapy (CPT-11 and CDDP). Gan To Kagaku Ryoho. 2010;37(8):1579-82.

23. Chen JL, Cheng JC, Kuo SH, Chen CA, Lin MC, Huang CY. Outcome analysis of cervical adenocarcinoma carcinoma compared with adenocarcinoma. Acta Obstet Gynecol Scand. 2012;91(10):1158-66.

24. Meng YH, Li S, Hu T, Ma D, Lu YP, Wang H. Clinical analysis of 132 cases of cervical adenosquamous carcinoma and cervical adenocarcinoma. Chin J Cancer. 2010;29(1):15-9. 